\title{
Tumor expression of environmental chemical-responsive genes and breast cancer mortality
}

\author{
Vasily N Aushev'1, Kalpana Gopalakrishnan'1, Susan L Teitelbaum¹, Humberto Parada Jr2, Regina M Santella³, \\ Marilie D Gammon4 and Jia Chen ${ }^{1,5,6,7}$ \\ 1Department of Environmental Medicine and Public Health, Icahn School of Medicine at Mount Sinai, New York, New York, USA \\ 2Division of Epidemiology \& Biostatistics, School of Public Health, San Diego State University, San Diego, California, USA \\ 32Department of Environmental Health Sciences, Columbia University, New York, New York, USA \\ ${ }^{4}$ University of North Carolina at Chapel Hill, Chapel Hill, North Carolina, USA \\ ${ }^{5}$ Department of Pediatrics, Icahn School of Medicine at Mount Sinai, New York, New York, USA \\ ${ }^{6}$ Department of Medicine, Hematology and Medical Oncology, Icahn School of Medicine at Mount Sinai, New York, New York, USA \\ ${ }^{7}$ Department of Oncological Sciences, Icahn School of Medicine at Mount Sinai, New York, New York, USA
}

Correspondence should be addressed to J Chen: jia.chen@mssm.edu

\begin{abstract}
Environmental phenols and phthalates are common ingredients in personal care products and some have been implicated in breast cancer progression. We have previously identified genes differentially expressed in response to low-dose exposure to diethyl phthalate (DEP) and methyl paraben (MPB) in a rat model. Herein we explore if these genes are associated with breast cancer mortality in humans. We profiled MPB- and DEP-responsive genes in tumors by NanoString $®$ from a population-based cohort of 606 women with first primary breast cancer among whom 119 breast cancer-specific deaths occurred within 15+ years of follow-up. For each gene, Cox proportional hazards models were used to estimate hazard ratios (HRs) and 95\% confidence intervals (Cls). Results were validated in two publicly available datasets. The following results were obtained. From 107 DEP- and 77 MPB-responsive genes profiled, 44 and 30 genes, respectively, were significantly associated with breast cancer-specific mortality. Some top DEPresponsive genes are novel for breast cancer mortality, such as $A B H D 14 B$ (for high-vs-low expression, HR 0.36, 95\% Cl: 0.2-0.5) and TMC4 (HR 0.37, 95\% Cl: 0.3-0.5); top hits for MPB (SLC4OA1 (HR 0.37, 95\% Cl: 0.3-0.5) and NTN4 (HR 0.39, 95\% Cl: 0.3-0.6)) are wellknown predictors of breast cancer survival. PLEKHA6 was another novel survival predictor, sensitive to hormonal receptor status (HR 0.5, 95\% Cl 0.3-0.9 for hormonal receptorpositive and HR 3.2, 95\% Cl 1.7-6.2 for -negative group). In conclusion, tumor expression of DEP- and MPB-responsive genes is associated with breast cancer mortality, supporting that exposure to these chemicals may influence the progression of breast cancer.
\end{abstract}

\section{Introduction}

Low-molecular-weight phthalates and parabens are two classes of environmental chemicals widely used in personal care and medical products as plasticizers and solvents
(C) 2019 Society for Endocrinology Published by Bioscientifica Ltd. Printed in Great Britain (phthalates) or preservatives (parabens). Phthalate-based additives are known to leach from the plastic matrix and can end up in the environment and in humans 
(Bosnir et al. 2003, Schettler 2006). Similarly, parabens, as antimicrobial agents, are directly added to food and pharmaceutical products (Soni et al. 2002). Many of these chemicals have shown to have endocrine-disruption properties which have the capacity of interfering the endocrine system and possibly playing a role in breast carcinogenesis (Delfosse et al. 2015). Humans are exposed to these chemicals through ingestion, inhalation and dermal contact. Neither phthalates nor parabens accumulate in the body and are rapidly enzymatically metabolized; phthalate diesters are hydrolyzed to the monoesters which are then absorbed and further oxidized (Albro \& Lavenhar 1989, Silva et al. 2003), while parabens are metabolized to 4-hydroxybenzoic acid (PHBA) (Soni et al. 2001, 2002, Lakeram et al. 2006). Low-to-no acute toxicity or mutagenicity has been reported so far (Matthews et al. 1956), but some studies indicated estrogenic or anti-estrogenic activity for at least some phthalates (Jobling et al. 1995, Harris et al. 1997). These data suggest a potential role of parabens and phthalates in the development or progression of breast cancer (Darbre \& Harvey 2014, Giulivo et al. 2016, Benjamin et al. 2017), but existing data on this subject are scarce, especially for humans.

Our study focuses on two specific phthalate and paraben congeners: diethyl phthalate (DEP, CAS \# 84-66-2) and methyl paraben (MPB, CAS \# 99-76-3) that are commonly used in personal care products. DEP is a low-molecular-weight phthalate widely used as a solvent in various consumer products, especially fragrance-containing ones (Api 2001). In the body, DEP is metabolized to mono-ethyl phthalate (MEP, CAS \# 2306-33-4) which is readily detectable in urine. A study of Mexican women found positive associations between the highest concentrations of MEP and breast cancer risk (Lopez-Carrillo et al. 2010). However, we did not observe such association in our population-based Long Island Breast Cancer Caner Study Project (LIBCSP) (Parada et al. 2018). MPB is used, similarly to other parabens, as an antimicrobial agent in cosmetics, foods and pharmaceutical products. As the shortest member of the parabens, MPB displays the highest ability to penetrate the skin (Cosmetic Ingredient Review 2008); its urinary metabolite is PHBA, thus indistinguishable from other parabens. While earlier studies assumed extremely weak estrogenic activity of all parabens (Golden et al. 2005), there are several recent works demonstrating estrogenic effects of parabens (Darbre \& Harvey 2014) including $\mathrm{MPB}$, in both in vitro (Pugazhendhi et al. 2005) and in vivo (Sun et al. 2016) settings. Importantly, many in vitro studies report carcinogenic effects of MPB (Wrobel \& Gregoraszczuk 2013, Khanna et al. 2014, Lillo et al. 2017); at the same time, our recent population study did not find significant correlations between urinary levels of MPB and breast cancer incidence or mortality (Parada et al. 2019).

In our previous studies of a rat model, we found that low-dose exposure to MPB and DEP at critical windows of development induced changes in histology and development of mammary glands (Manservisi et al. 2015) and further identified a gene signature of exposure to low-dose DEP and MPB in the rat mammary glands: a set of genes was shown to be differentially expressed in rats exposed to low doses of MPB (Gopalakrishnan et al. 2017) and DEP (Gopalakrishnan K, Aushev VN, Teitelbaum SL, Manservisi F, Falcioni L, Panzacchi S, Belpoggi F, Parada H Jr, Garbowski G, Hibshoosh H, Santella RM, Gammon MD \& Chen J, unpublished observations). In the current study, we aimed to examine whether the expression of these chemical-responsive genes in breast cancer tissue was associated with breast cancer mortality in a populationbased study.

\section{Materials and methods}

\section{Study population}

We utilized resources from the follow-up component of the LIBCSP, which includes a population-based cohort of women newly diagnosed with first primary breast cancer who participated in the baseline interview within 2 to 3 months of diagnosis (Gammon et al. 2002) and were subsequently re-interviewed about 5 years later and followed for vital status (Cleveland et al. 2007). Participants were 1508 women newly diagnosed with first primary breast cancer between August 1, 1996 and July 31, 1997, who resided in Nassau and Suffolk counties of Long Island, NY. LIBCSP participants were predominantly (93\%) Caucasian, and aged 25-98 (median age 58 years). Potentially eligible women were identified through daily or weekly contact with pathology/cytology departments of 33 collaborating institutions and confirmed by medical records review. The National Death Index (NDI) was used to ascertain all-cause and breast cancer-specific mortality among study participants, who were followed from diagnosis until December 31, 2014, for an average of 162 months (range 2.7-224 months, median 213 months) for the women included in this study. We obtained sufficient RNA for profiling from paraffin-embedded 
tumor tissue from 616 of the 1508 patients; for the other LIBCSP patients, tumor slides were not made available by the diagnosing hospitals $(n=763)$ or the extracted RNA was of insufficient/low quality $(n=139)$; of these, 606 passed further QC. Demographics of both the overall patient cohort and the subset with sufficient tissue RNA are presented in a previously published study (Aushev et al. 2017). Among the 606 women with successfully profiled tumor tissue, 275 deaths occurred by the end of the follow-up period, of which 119 (43\%) were related to breast cancer (ICD codes 174.9 and C-50.9 listed as a primary or secondary code on the death certificate). Consent has been obtained from each patient or subject after full explanation of the purpose and nature of all procedures used. The study protocol was approved by the Institutional Review Boards of the collaborating institutions.

\section{Selection of MPB- and DEP-responsive genes}

Discovery of MPB-responsive genes was previously published by Gopalakrishnan et al. (2017). Briefly, the MPB-responsive gene signature was identified based on differentially expressed genes in Sprague- Dawley rat mammary glands following low-dose MPB exposure during different windows of development. For pubertal MPB exposure, 295 genes were differentially expressed; after bootstrapping the samples and finding human orthologs, 77 genes ( 42 downregulated and 35 upregulated by MPB) were selected for further analysis in LIBCSP samples. Similarly, in DEP-exposed parous and nulliparous rats, 107 genes were selected as significantly differentially expressed (Gopalakrishnan K, Aushev VN, Teitelbaum SL, Manservisi F, Falcioni L, Panzacchi S, Belpoggi F, Parada H Jr, Garbowski G, Hibshoosh H, Santella RM, Gammon MD \& Chen J, unpublished observations); importantly, all but one of these genes were downregulated by DEP exposure.

\section{Tissue processing and RNA extraction}

Formalin-fixed paraffin-embedded (FFPE) tumor sections from the population-based cohort were histopathologically reviewed by a trained pathologist and the cancer tissue was separated using manual microdissection (Rossner et al. 2009). Total RNA was extracted from tumor tissues using the Qiagen miRNeasy FFPE kit (Qiagen). RNA concentration and quality were determined with a NanoDrop spectrophotometer (Thermo Scientific). In total, 606 samples with sufficient RNA concentration were used in our analyses.

C 2019 Society for Endocrinology Published by Bioscientifica Ltd. Printed in Great Britain

\section{Gene expression profiling}

Gene expression analysis of MPB- and DEP-responsive genes was performed with the NanoString nCounter platform (Seattle, WA, USA), using a custom-designed codeset and following the manufacturer's instructions. Briefly, 100 ng RNA was incubated with reporter and capture probes overnight at $65^{\circ} \mathrm{C}$. Following hybridization, unbound probes were removed, and the purified complexes were aligned and immobilized on imaging cartridges using an nCounter Prep station. Code count detection was carried out by scanning cartridges in an nCounter Digital Analyzer to determine gene expression levels. As a quality control filter, we excluded all samples having less than 50,000 total counts for positive probes, or having less than $1.5 \times$ blank counts for housekeeping and endogenous genes. Raw counts were imported from RCC files with the NanoStringQCPro R package (version 1.6.0), background was subtracted as mean of negative control counts, geometric mean of housekeeping genes counts was used for normalization, and values were logtransformed for further analyses.

Coexpression of profiled genes was analyzed in $\mathrm{R}$ by building a covariance matrix; and principal component analysis was performed with 'pca' function of 'pcaMethods' package (Stacklies et al. 2007).

\section{Molecular characteristics of tumors}

Molecular characterization of the tumor tissues used in the current study is described in Aushev et al. (2017). Briefly, we determined hormone receptor status by measuring the expression of estrogen receptor (ER) and progesterone receptor (PR) genes, which was highly correlated with hormone receptor status determined by immunohistochemistry. Expression of the PAM50 panel of genes (Parker et al. 2009) was measured within the same NanoString panel and risk-of-recurrence score was determined as previously described in Aushev et al. (2017).

\section{Survival analysis}

Cox proportional hazards models (Cox 1972) were used for estimating the multivariable-adjusted hazard ratios (HRs) and 95\% confidence intervals (CIs) for breast cancer-specific mortality associated with gene expression among the 606 women in our population-based cohort; non-breast cancer-specific deaths were censored. Survival analysis was performed using 'survival' package 
in R (Borgan 2001). For each gene, range of cutoff thresholds (from second to eighth decile) was tested and the threshold with the lowest $P$ value was used (Mihaly et al. 2013). For each gene, a Cox model was built using 'coxph' function with age and tumor stage (invasive or in situ) included as covariates. Multiple comparison adjustment was applied, where indicated, with the false discovery rate (FDR) threshold at 0.05 .

All models were initially adjusted for age at diagnosis (continuous) and tumor stage. We further evaluated potential confounding using the methods described by Rothman and Greenland (Rothman et al. 2008) starting with a full multivariate model and using backward elimination. Potential confounders considered included: menopausal status (pre-/post-menopausal), family history of breast cancer in a first-degree relative, BMI at diagnosis, tumor size and education. If eliminating a covariate from the full Cox regression model changed the effect estimate by $10 \%$ or more, the covariate was considered a confounder and kept in the model. Otherwise that covariate was dropped from the multivariate model. None of the covariates tested met this criterion; thus, only results adjusted for age and tumor stage were presented. We also performed analyses stratified by ER/PR status (cases positive by the expression of at least one of the receptors, hereafter named as $\mathrm{ER} \mid \mathrm{PR}+\mathrm{vs}$ cases negative by both receptors, hereafter named as ER-/PR-). KaplanMeier survival (Kaplan \& Meier 1958) plots were built for visualization of unadjusted associations between gene expression and breast cancer-specific mortality.

\section{Results}

From 77 MPB-responsive genes (42 downregulated and 35 upregulated by MPB) profiled in tumors, 30 genes (39\%) were significantly (FDR $\leq 0.05$ ) associated with breast cancer-specific survival (Fig. 1 and Supplementary Table 1 , see section on supplementary data given at the end of this article). Increased expression of 17 of these genes was associated with lower breast cancer mortality, that is, longer survival. Strongest associations were found for SLC4OA1 (Ferroportin-1) (HR 0.4, 95\% CI: 0.3-0.5) and NTN4 (Netrin-4) (HR 0.4, 95\% CI: 0.3-0.6), both are known breast cancer onco-suppressor genes. From those 17 genes associated with reduced breast cancer mortality, ten were downregulated by MPB in rats. For 13 remaining MPB-responsive genes, inverse associations were found, that is, their increased expression was associated with higher cancer mortality
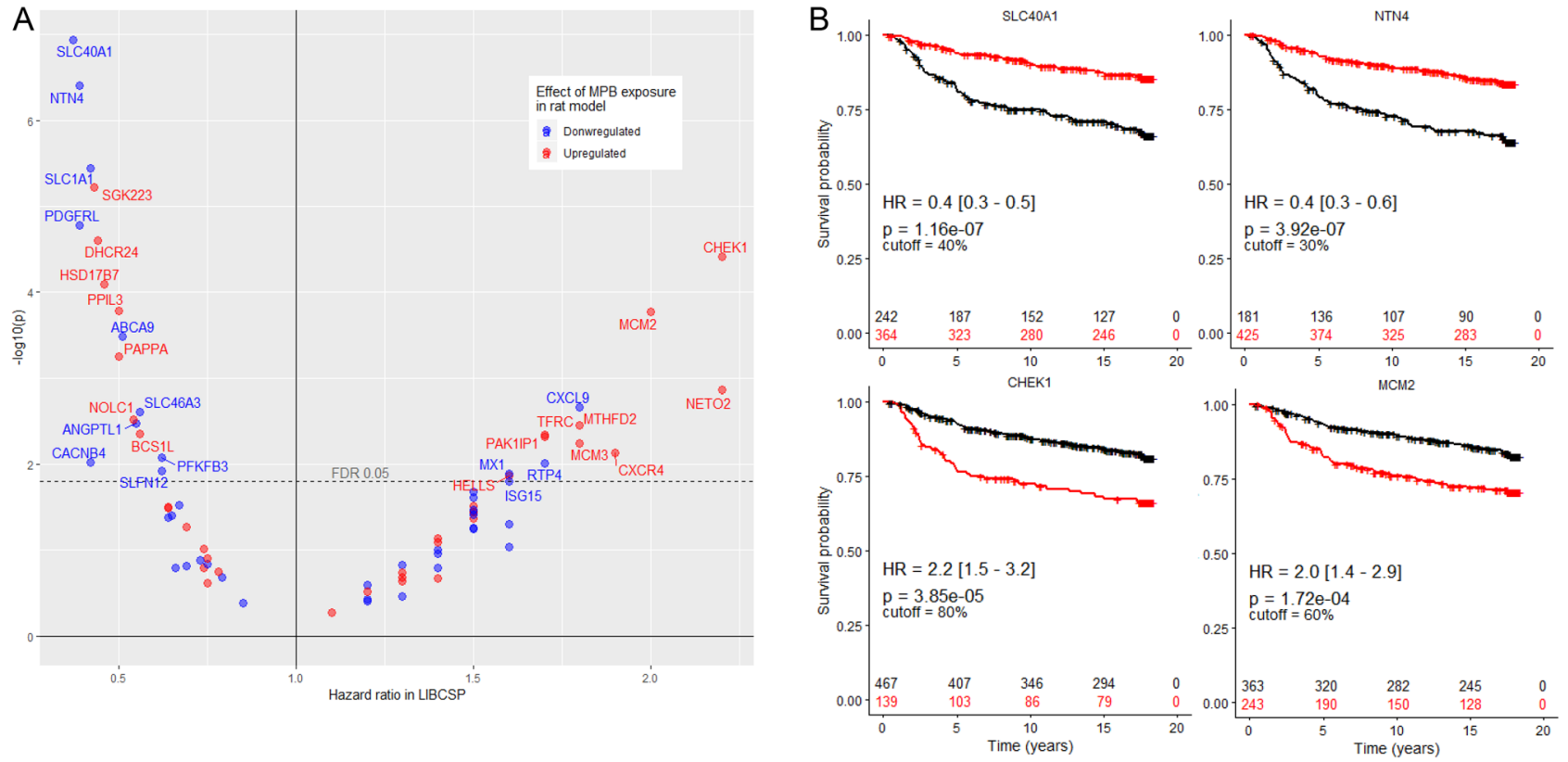

\section{Figure 1}

Correlation of expression of 77 MPB-related genes with long-term breast cancer-specific survival of LIBCSP patients. (A) Volcano plot, showing age- and stage-adjusted Cox models, with each dot corresponding to a gene, horizontal axis displaying hazard ratio of high expression group comparing to low expression group (i.e. for the genes on the left higher expression is associated with better survival, genes on the right are associated with worse survival) and vertical axis displaying logarithm of $P$ value of the given Cox model. (B) Survival curves for representative genes associated with longer (SLC40A1, NTN4) and shorter (CHEK1, MCM2) survival. A full colour version of this figure is available at https://doi.org/10.1530/ERC-19-0357. 
(shorter survival), including established oncogenes CHEK1 (HR 2.2, 95\% CI: 1.5-3.2) and MCM2 (HR 2.0, 95\% CI: 1.4-2.9). Nine out of those 13 genes were upregulated by MPB in our rodent model.

Out of the 107 DEP-responsive genes tested (all but one of them were downregulated on DEP exposure), 44 genes $(41 \%)$ were significantly (FDR $\leq 0.05$ ) associated with breast cancer mortality (Fig. 2 and Supplementary Table 2). From these 44 genes, 34 of them were associated with lower mortality, with prolactin receptor $(P R L R)$, a known prognosis factor for low mortality, having the strongest association (for high-vs-low expression, HR 0.3, 95\% CI: 0.2-0.5) with breast cancer-specific survival. Importantly, two other top hits have never been reported to be linked to breast cancer or progression: Abhydrolase Domain Containing 14B (ABHD14B) (HR 0.4, 95\% CI: 0.2-0.5) and Transmembrane Channel Like 4 (TMC4) (HR 0.4, 95\% CI: 0.3-0.5).

From the 606 tumor samples profiled, 80\% were positive for ER or PR expression, as previously reported (Aushev et al. 2017). When the analyses were stratified by ER/PR status, it revealed that effects of some genes on breast cancer mortality were specific to the hormonal receptor status of the tumor. While the larger $(n=486)$ hormone receptor-positive $(\mathrm{ER} \mid \mathrm{PR}+)$ group revealed largely the same survival-associated genes found for the global population, in the hormone receptor negative $\left(\mathrm{ER}^{-} / \mathrm{PR}^{-}\right)$group $(n=120)$, only two DEP genes $(M X 1$, PLEKHA6) were strongly associated with breast cancer

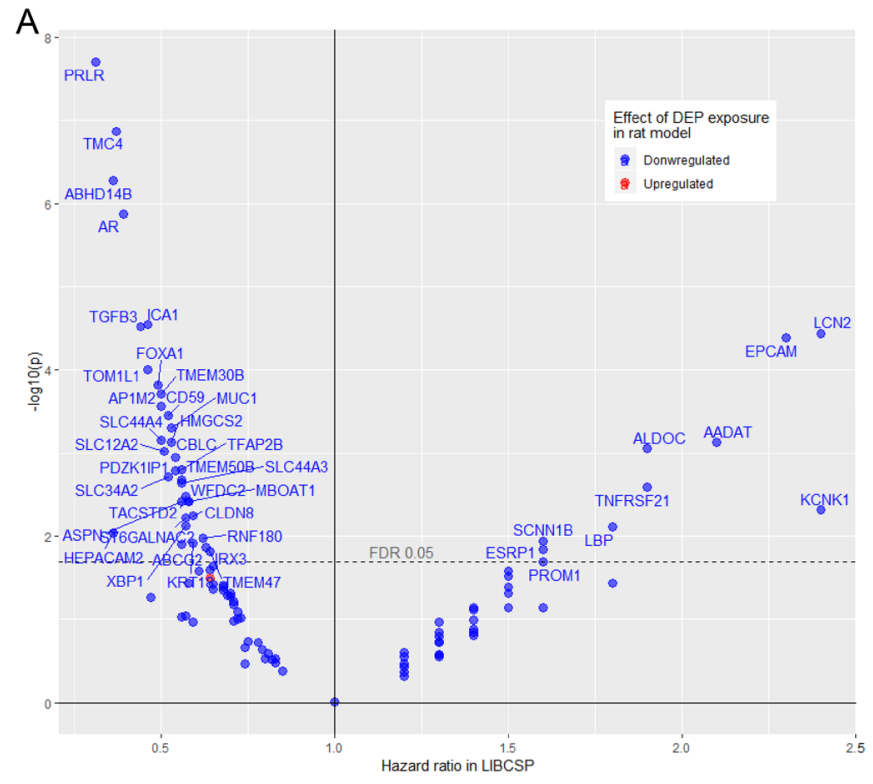

survival (FDR $\leq 0.05$ ): reduced mortality for MX1 (HR 0.3, 95\% CI: 0.2-0.6) and increased mortality for PLEKHA6 (HR 3.2, 95\% CI: 1.7-6.2). Interestingly, associations of these genes with breast cancer survival demonstrated opposite trends in ER|PR+ group: strongly increased mortality on higher expression of MX1 (HR 2.5, 95\% CI: 1.6-3.9) and reduced mortality for PLEKHA6 (HR 0.5, 95\% CI: 0.3-0.9) (Fig. 3).

Besides ER/PR status, we were also able to measure expression of the genes from the PAM50 panel (Parker et al. 2009) and thus assess distribution of molecular subtypes in analyzed samples and corresponding survival (see Supplementary Fig. 5 of the previously published work; Aushev et al. 2017). The most common was Luminal A subtype $(n=179)$, followed by Luminal B $(n=147)$, Basal $(n=114)$, Normal-like $(n=86)$ and Her2-enriched $(n=80)$. Because of low mortality in Luminal A and Normal-like groups (13 and 10 cases of breast cancer-associated deaths, respectively), we did not have enough statistical power to identify significant survival-associated genes in these groups separately, though the trends were similar. Thus, we combined Luminal A and Normal-like subgroups and confirmed the association for the top genes (TMC4: HR 0.2, 95\% CI 0.1-0.5; MX1: HR 3.8, 95\%CI 1.7-8.6; IRF7: HR 4.1, 95\% CI 1.8-9.3). Although other subtypes, such as Basal, and Luminal B, displayed higher mortality, they constitute less common breast cancer subtypes. After adjustment for multiple comparison, only in the Her2enriched subtype we identified two survival-related genes:

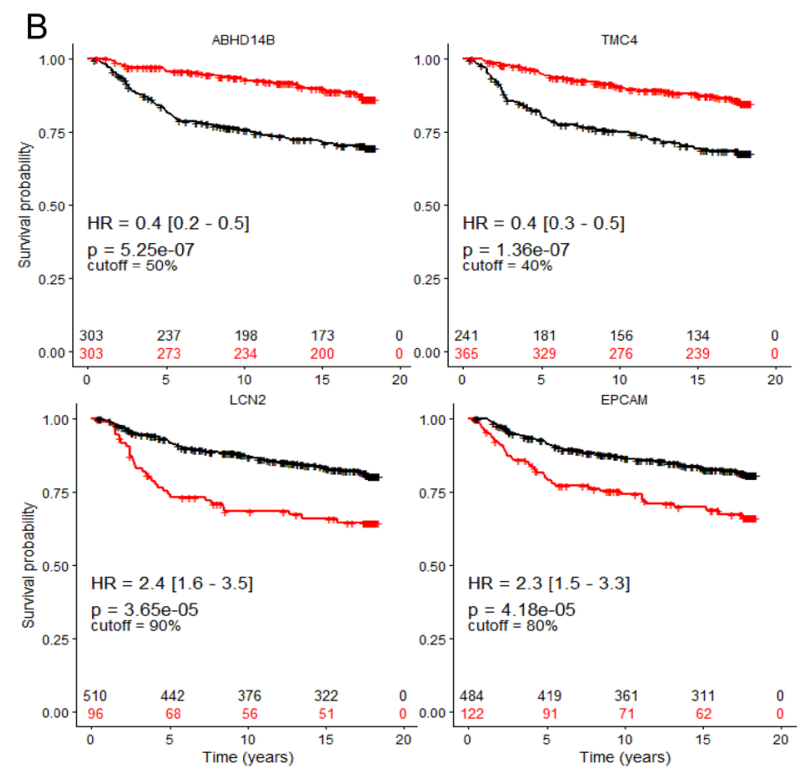

Figure 2

Correlation of expression of 107 DEP-related genes with breast cancer-specific survival; for legend see Fig. 1. A full colour version of this figure is available at https://doi.org/10.1530/ERC-19-0357. 

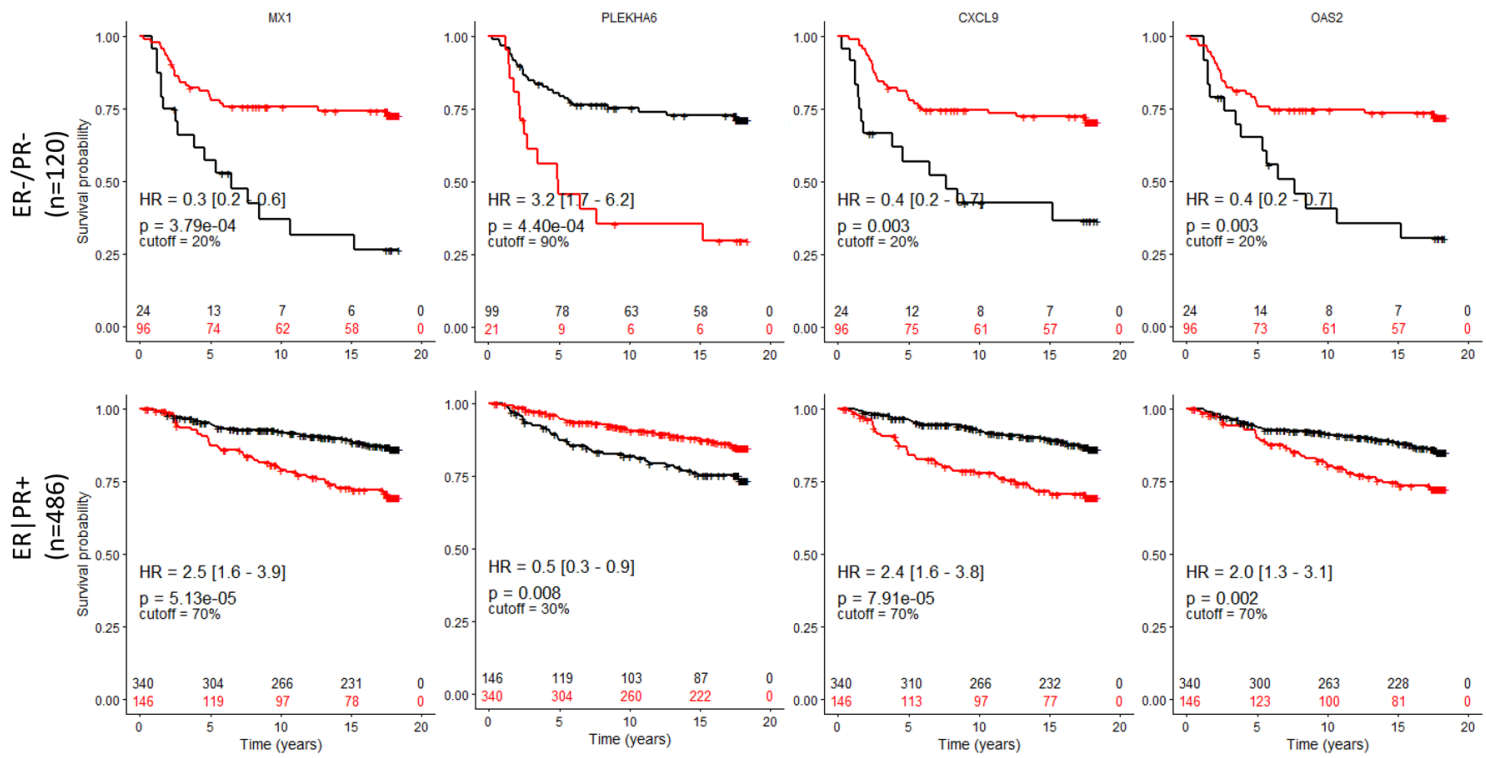

\section{Figure 3}

Survival-associated genes specific for hormonal receptor (ER/PR) expression status. Four representative genes shown, data for all genes are in the Supplementary Table 3. A full colour version of this figure is available at https://doi.org/10.1530/ERC-19-0357.

EPCAM (HR 4.2, 95\% CI: 2.0-8.9) and QSOX1 (HR 4.2, 95\% CI: 2.0-9.0). Complete results of survival analysis for all groups are available in Supplementary Table 3.

Using the PAM50 information and the algorithm (Parker et al. 2009) similar to the one used in clinical practice (Nielsen et al. 2014), we have estimated the risk-of-recurrence score for each patient of the LIBCSP cohort in our previous study (Aushev et al. 2017). This allowed us to re-analyze survival association of our genes of interest after stratification for the high- $(n=249)$ and low/medium- $(n=357)$ risk-of-recurrence groups. For the genes that co-expressed with the markers from PAM50 panels, we expected weaker association after this stratification. However, associations of top hits such as TMC4 and SLC4OA1 remained significant in the low/medium-risk group: patients with low expression of these genes had high risk of recurrence despite being classified as low/medium risk by the conventional panel (Supplementary Fig. 2). For the PAM50-high-risk group, PPAP2C gene displayed the strongest association (HR 2.6, 95\% CI: 1.7-4.1).

To validate our findings, we performed a similar survival analysis using two publicly available data sources - METABRIC and KMplot. The METABRIC dataset (Curtis et al. 2012) includes 2000 breast cancer cases, with demographic and clinical characteristics similar to those of LIBCSP (predominantly Caucasian, 77\% ER+). KMplot service (Gyorffy et al. 2010) combines multiple independent datasets including more than 1700 breast cancer cases.
As shown in Supplementary Fig. 1A, our most significant associations were reproducible in both METABRIC and KMplot datasets: genes correlated with better survival such as SLC4OA1, NTN, ABHD14B - all remained significantly associated and with the same direction of association. ER/PR-status-specific associations were also largely confirmed: higher expression of MX1 was associated with higher mortality in ER/PR-positive subgroup for all three datasets but lower mortality in ER/PR-negative subgroup (not significant in METABRIC dataset); the opposite association for PLEKHA6 was confirmed in KMPlot data (not significant in METABRIC) (Supplementary Fig. 1B). Supplementary Table 4 contains survival analysis data for the genes of interest available at METABRIC.

\section{Discussion}

In this study, we assessed the expression of MPB- and DEPresponsive genes, for their association with breast cancerspecific mortality. These genes were selected based on our previous works on animal model where we studied gene expression in mammary gland after the corresponding exposure. In the current work, we investigated associations between expression of these candidate genes in human breast tumors, with breast cancer mortality. We observed significant associations for $\sim 40 \%$ of the genes examined. In both MPB and DEP gene sets, for the majority of the genes their higher expression was associated with increased c) 2019 Society for Endocrinology Published by Bioscientifica Ltd. Printed in Great Britain 
mortality. These associations were also largely confirmed in two independent datasets, METABRIC and KMplot.

The possible role of ED chemicals in breast cancer initiation and progression remains debatable. For MPB, early studies did not show a carcinogenic effect (Golden et al. 2005), and the estrogenic activity of parabens is considered extremely weak (Golden et al. 2005). For phthalates, estrogenic, anti-estrogenic or androgenic effects were reported in in vitro and animal studies but the results are often inconsistent and mostly obtained with high-dose exposure (Lovekamp-Swan \& Davis 2003, Mckee et al. 2004). Our recent report did not show significant association between urinary phthalate levels and breast cancer incidence nor mortality (Parada et al. 2018). For parabens on the other hand, inverse associations with borderline significance was found (HR for $\operatorname{Ln}$ (methylparaben concentration): 0.92, 95\% CI: 0.85-1.00, $P=0.05$; HR for Ln(propylparaben concentration): 0.93, 95\% CI: 0.87-0.99, $P=0.03$ ) (Parada et al. 2019). In our previous animal study, we found that low-dose DEP and MPB exposure during certain windows of development induced significant morphological/ histological and transcriptome changes in rat mammary glands (Manservisi et al. 2015, Gopalakrishnan et al. 2017). Our current study demonstrates that most of the genes that are, in a rat model, shown to be downregulated by DEP exposure (Gopalakrishnan et al. 2017) are associated with reduced breast cancer mortality (PRLR, TMC4, $A B H D 14 B, A R$, and others - see Fig. 2); these results are consistent with the 'oncogenic' effect of phthalate. For $\mathrm{MPB}$, a similar trend is observed: MPB-downregulated SLC4OA1, NTN4, SLC1A1, PDGFRL, and ABCA9 are all mortality reducing, while MPB-upregulated CHEK1 and MCM2 are mortality enhancing (Fig. 1). However, a small number of genes showed the opposite trend including MPB-upregulated SGK223, DHCR24, HSD17B7, and PPIL3 and DEP-downregulated LCN2, EPCAM, ALDOC, and $A A D A T$. Therefore, we cannot make an unequivocal conclusion about oncogenic or onco-protective roles for these chemicals, reflecting the complex nature of environmental influence on breast carcinogenesis.

To our knowledge, this is the first study establishing a link between DEP- and MPB-responsive genes, and survival of breast cancer patients. We acknowledge certain limitations of this study. As in any translational research, results originated from animal experiments cannot be fully translated to humans; for the same reason, it is not surprising that not all the genes were concordant between the two characteristics we were interested in: association with survival on one hand and association with
MPB/DEP exposure on the other. Additional human studies are needed to assess this link more directly. At the same time, despite the limitation of animal-to-human translation, our findings on the association of these genes with breast cancer mortality, especially confirmed by two large independent datasets, are informative to the underlying mechanism. It is important to understand, however, that our observations are limited to certain types of tumors and/or populations. LIBCSP population consists of predominantly women of European ancestry, with $80 \%$ cases being hormonal receptor positive; all the strongest associations we found for this population overall did stand significant for ER/PR-positive subgroup, but not for the smaller subgroup of ER/PR-negative cases where other genes were revealed as strong predictors.

In terms of practical significance, it is important to mark the genes that allowed refining cases 'misclassified' by the conventional PAM50 panel: for example, TMC4 alone was able to distinguish high-risk patients that were classified as low or medium risk.

Overall, we believe that our findings shed light on possible mechanism and identify previously unknown players in breast carcinogenesis and novel predictors of breast cancer mortality, which may potentially be valuable for clinical practice.

\section{Supplementary data}

This is linked to the online version of the paper at https://doi.org/10.1530/ ERC-19-0357.

\section{Declaration of interest}

The authors declare that there is no conflict of interest that could be perceived as prejudicing the impartiality of the research reported.

\section{Funding}

This work was supported by grant from the National Institutes of Health (NIH R01 CA172460) and in part by grants 5U01 ES019459, UO1CA/ES66572.

Ethics approval and consent to participate

For the Long Island Breast Cancer Study Project (LIBCSP), Institutional Review Board approval was obtained by all participating institutions and informed consent was obtained from all study participants; study was approved by the Institutional Review Board of Mount Sinai (Program for the Protection of Human Subjects).

\section{References}

Albro PW \& Lavenhar SR 1989 Metabolism of di(2-ethylhexyl)phthalate. Drug Metabolism Reviews 21 13-34. (https://doi. org/10.3109/03602538909029953) (c) 2019 Society for Endocrinology Published by Bioscientifica Ltd. Printed in Great Britain 
Api AM 2001 Toxicological profile of diethyl phthalate: a vehicle for fragrance and cosmetic ingredients. Food and Chemical Toxicology 39 97-108. (https://doi.org/10.1016/s0278-6915(00)00124-1)

Aushev VN, Lee E, Zhu J, Gopalakrishnan K, Li Q, Teitelbaum SL, Wetmur JG, Degli Esposti D, Hernandez-Vargas H, Herceg Z, et al. 2017 Novel predictors of breast cancer survival derived from mirna activity analysis. Clinical Cancer Research 24 581-591. (https://doi. org/10.1158/1078-0432.CCR-17-0996)

Benjamin S, Masai E, Kamimura N, Takahashi K, Anderson RC \& Faisal PA 2017 Phthalates impact human health: epidemiological evidences and plausible mechanism of action. Journal of Hazardous Materials 340 360-383. (https://doi.org/10.1016/j. jhazmat.2017.06.036)

Borgan Ø 2001 Modeling survival data: extending the cox model. Terry M. Therneau and Patricia M. Grambsch, Springer-Verlag, New York, 2000. Statistics in Medicine 20 2053-2054.

Bosnir J, Puntaric D, Skes I, Klaric M, Simic S \& Zoric I 2003 Migration of phthalates from plastic products to model solutions. Collegium Antropologicum 27 (Supplement 1) 23-30.

Cleveland RJ, Eng SM, Abrahamson PE, Britton JA, Teitelbaum SL, Neugut AI \& Gammon MD 2007 Weight gain prior to diagnosis and survival from breast cancer. Cancer Epidemiology, Biomarkers and Prevention 16 1803-1811. (https://doi.org/10.1158/1055-9965.EPI-060889)

Cosmetic ingredient Review 2008 Final amended report on the safety assessment of methylparaben, ethylparaben, propylparaben, isopropylparaben, butylparaben, isobutylparaben, and benzylparaben as used in cosmetic products. International Journal of Toxicology $\mathbf{2 7}$ (Supplement 4) 1-82.

Cox DR 1972 Regression models and life-tables. Journal of the Royal Statistical Society: Series B 34 187-202. (https://doi. org/10.1111/j.2517-6161.1972.tb00899.x)

Curtis C, Shah SP, Chin SF, Turashvili G, Rueda OM, Dunning MJ, Speed D, Lynch AG, Samarajiwa S, Yuan Y, et al. 2012 The genomic and transcriptomic architecture of 2000 breast tumours reveals novel subgroups. Nature 486 346-352. (https://doi.org/10.1038/ nature10983)

Darbre PD \& Harvey PW 2014 Parabens can enable hallmarks and characteristics of cancer in human breast epithelial cells: a review of the literature with reference to new exposure data and regulatory status. Journal of Applied Toxicology 34 925-938. (https://doi. org/10.1002/jat.3027)

Delfosse V, Maire AL, Balaguer P \& Bourguet W 2015 A structural perspective on nuclear receptors as targets of environmental compounds. Acta Pharmacologica Sinica 36 88-101. (https://doi. org/10.1038/aps.2014.133)

Gammon MD, Neugut AI, Santella RM, Teitelbaum SL, Britton JA, Terry MB, Eng SM, Wolff MS, Stellman SD, Kabat GC, et al. 2002 The Long Island Breast Cancer Study Project: description of a multiinstitutional collaboration to identify environmental risk factors for breast cancer. Breast Cancer Research and Treatment 74 235-254. (https://doi.org/10.1023/a:1016387020854)

Giulivo M, Lopez De Alda M, Capri E \& Barcelo D 2016 Human exposure to endocrine disrupting compounds: their role in reproductive systems, metabolic syndrome and breast cancer. A review. Environmental Research 151 251-264. (https://doi. org/10.1016/j.envres.2016.07.011)

Golden R, Gandy J \& Vollmer G 2005 A review of the endocrine activity of parabens and implications for potential risks to human health. Critical Reviews in Toxicology 35 435-458. (https://doi. org/10.1080/10408440490920104)

Gopalakrishnan K, Teitelbaum SL, Lambertini L, Wetmur J, Manservisi F, Falcioni L, Panzacchi S, Belpoggi F \& Chen J 2017 Changes in mammary histology and transcriptome profiles by low-dose exposure to environmental phenols at critical windows of development.
Environmental Research 152 233-243. (https://doi.org/10.1016/j. envres.2016.10.021)

Gyorffy B, Lanczky A, Eklund AC, Denkert C, Budczies J, Li Q \& Szallasi Z 2010 An online survival analysis tool to rapidly assess the effect of 22,277 genes on breast cancer prognosis using microarray data of 1809 patients. Breast Cancer Research and Treatment 123 725731. (https://doi.org/10.1007/s10549-009-0674-9)

Harris CA, Henttu P, Parker MG \& Sumpter JP 1997 The estrogenic activity of phthalate esters in vitro. Environmental Health Perspectives 105 802-811. (https://doi.org/10.1289/ehp.97105802)

Jobling S, Reynolds T, White R, Parker MG \& Sumpter JP 1995 A variety of environmentally persistent chemicals, including some phthalate plasticizers, are weakly estrogenic. Environmental Health Perspectives 103 582-587. (https://doi.org/10.1289/ehp.95103582)

Kaplan EL \& Meier P 1958 Nonparametric estimation from incomplete observations. Journal of the American Statistical Association $\mathbf{5 3} 457-$ 481. (https://doi.org/10.1080/01621459.1958.10501452)

Khanna S, Dash PR \& Darbre PD 2014 Exposure to parabens at the concentration of maximal proliferative response increases migratory and invasive activity of human breast cancer cells in vitro. Journal of Applied Toxicology 34 1051-1059. (https://doi.org/10.1002/jat.3003)

Lakeram M, Paine AJ, Lockley DJ, Sanders DJ, Pendlington R \& Forbes B 2006 Transesterification of p-hydroxybenzoate esters (parabens) by human intestinal (Caco-2) cells. Xenobiotica 36 739-749. (https://doi. org/10.1080/00498250600738637)

Lillo MA, Nichols C, Perry C, Runke S, Krutilina R, Seagroves TN, Miranda-Carboni GA \& Krum SA 2017 Methylparaben stimulates tumor initiating cells in ER+ breast cancer models. Journal of Applied Toxicology 37 417-425. (https://doi.org/10.1002/jat.3374)

Lopez-Carrillo L, Hernandez-Ramirez RU, Calafat AM, Torres-Sanchez L, Galvan-Portillo M, Needham LL, Ruiz-Ramos R \& Cebrián ME 2010 Exposure to phthalates and breast cancer risk in northern Mexico. Environmental Health Perspectives 118 539-544. (https://doi. org/10.1289/ehp.0901091)

Lovekamp-Swan T \& Davis BJ 2003 Mechanisms of phthalate ester toxicity in the female reproductive system. Environmental Health Perspectives 111 139-145. (https://doi.org/10.1289/ehp.5658)

Manservisi F, Gopalakrishnan K, Tibaldi E, Hysi A, Iezzi M, Lambertini L, Teitelbaum S, Chen J \& Belpoggi F 2015 Effect of maternal exposure to endocrine disrupting chemicals on reproduction and mammary gland development in female Sprague-Dawley rats. Reproductive Toxicology $\mathbf{5 4}$ 110-119. (https://doi.org/10.1016/j.reprotox.2014.12.013)

Matthews C, Davidson J, Bauer E, Morrison JL \& Richardson AP 1956 P-Hydroxybenzoic acid esters as preservatives. II. Acute and chronic toxicity in dogs, rats, and mice. Journal of the American Pharmaceutical Association 45 260-267. (https://doi.org/10.1002/ jps.3030450420)

Mckee RH, Butala JH, David RM \& Gans G 2004 NTP center for the evaluation of risks to human reproduction reports on phthalates: addressing the data gaps. Reproductive Toxicology 18 1-22. (https:// doi.org/10.1016/j.reprotox.2003.09.002)

Mihaly Z, Kormos M, Lanczky A, Dank M, Budczies J, Szasz MA \& Gyorffy B 2013 A meta-analysis of gene expression-based biomarkers predicting outcome after tamoxifen treatment in breast cancer. Breast Cancer Research and Treatment 140 219-232. (https://doi. org/10.1007/s10549-013-2622-y)

Nielsen T, Wallden B, Schaper C, Ferree S, Liu S, Gao D, Barry G, Dowidar N, Maysuria M \& Storhoff J 2014 Analytical validation of the PAM50-based Prosigna Breast Cancer Prognostic Gene Signature Assay and nCounter Analysis System using formalin-fixed paraffinembedded breast tumor specimens. BMC Cancer 14 177. (https://doi. org/10.1186/1471-2407-14-177)

Parada Jr H, Gammon MD, Chen J, Calafat AM, Neugut AI, Santella RM, Wolff MS \& Teitelbaum SL 2018 Urinary phthalate metabolite concentrations and breast cancer incidence and survival following https://erc bioscientifica com

https://doi.org/10.1530/ERC-19-0357
C) 2019 Society for Endocrinology Published by Bioscientifica Ltd. Printed in Great Britain 
breast cancer: the Long Island Breast Cancer Study Project. Environmental Health Perspectives 126 047013. (https://doi. org/10.1289/EHP2083)

Parada Jr H, Gammon MD, Ettore HL, Chen J, Calafat AM, Neugut AI, Santella RM, Wolff MS \& Teitelbaum SL 2019 Urinary concentrations of environmental phenols and their associations with breast cancer incidence and mortality following breast cancer. Environment International 130 104890. (https://doi.org/10.1016/j.envint.2019.05.084)

Parker JS, Mullins M, Cheang MC, Leung S, Voduc D, Vickery T, Davies S, Fauron C, He X, Hu Z, et al. 2009 Supervised risk predictor of breast cancer based on intrinsic subtypes. Journal of Clinical Oncology 27 1160-1167. (https://doi.org/10.1200/JCO.2008.18.1370)

Pugazhendhi D, Pope GS \& Darbre PD 2005 Oestrogenic activity of p-hydroxybenzoic acid (common metabolite of paraben esters) and methylparaben in human breast cancer cell lines. Journal of Applied Toxicology 25 301-309. (https://doi.org/10.1002/jat.1066)

Rossner Jr P, Gammon MD, Zhang YJ, Terry MB, Hibshoosh H, Memeo L, Mansukhani M, Long CM, Garbowski G, Agrawal M, et al. 2009 Mutations in p53, p53 protein overexpression and breast cancer survival. Journal of Cellular and Molecular Medicine 13 38473857. (https://doi.org/10.1111/j.1582-4934.2008.00553.x)

Rothman KJ, Greenland S \& Lash TL 2008 Modern Epidemiology. Philadelphia, PA, USA: Wolters Kluwer Health/Lippincott Williams \& Wilkins.

Schettler T 2006 Human exposure to phthalates via consumer products. International Journal of Andrology 29 134-139; discussion 181-185. (https://doi.org/10.1111/j.1365-2605.2005.00567.x)
Silva MJ, Barr DB, Reidy JA, Kato K, Malek NA, Hodge CC, Hurtz 3rd D, Calafat AM, Needham LL \& Brock JW 2003 Glucuronidation patterns of common urinary and serum monoester phthalate metabolites. Archives of Toxicology 77 561-567. (https://doi. org/10.1007/s00204-003-0486-3)

Soni MG, Burdock GA, Taylor SL \& Greenberg NA 2001 Safety assessment of propyl paraben: a review of the published literature. Food and Chemical Toxicology 39 513-532. (https://doi.org/10.1016/ s0278-6915(00)00162-9)

Soni MG, Taylor SL, Greenberg NA \& Burdock GA 2002 Evaluation of the health aspects of methyl paraben: a review of the published literature. Food and Chemical Toxicology 40 1335-1373. (https://doi. org/10.1016/s0278-6915(02)00107-2)

Stacklies W, Redestig H, Scholz M, Walther D \& Selbig J 2007 pcaMethods - a bioconductor package providing PCA methods for incomplete data. Bioinformatics 23 1164-1167. (https://doi. org/10.1093/bioinformatics/btm069)

Sun L, Yu T, Guo J, Zhang Z, Hu Y, Xiao X, Sun Y, Xiao H, Li J, Zhu D, et al. 2016 The estrogenicity of methylparaben and ethylparaben at doses close to the acceptable daily intake in immature SpragueDawley rats. Scientific Reports 6 25173. (https://doi.org/10.1038/ srep25173)

Wrobel A \& Gregoraszczuk EŁ 2013 Effects of single and repeated in vitro exposure of three forms of parabens, methyl-, butyl- and propylparabens on the proliferation and estradiol secretion in MCF-7 and MCF-10A cells. Pharmacological Reports 65 484-493. (https://doi. org/10.1016/S1734-1140(13)71024-7)

Received in final form 5 September 2019

Accepted 7 October 2019

Accepted Preprint published online 8 October 2019 (c) 2019 Society for Endocrinology Published by Bioscientifica Ltd. Printed in Great Britain 